MATHEMATICS OF COMPUTATION

Volume 70, Number 233, Pages 307-318

S 0025-5718(00)01251-5

Article electronically published on March 16, 2000

\title{
A NEW CLASS OF RADIAL BASIS FUNCTIONS WITH COMPACT SUPPORT
}

\author{
M. D. BUHMANN
}

\begin{abstract}
Radial basis functions are well-known and successful tools for the interpolation of data in many dimensions. Several radial basis functions of compact support that give rise to nonsingular interpolation problems have been proposed, and in this paper we study a new, larger class of smooth radial functions of compact support which contains other compactly supported ones that were proposed earlier in the literature.
\end{abstract}

\section{INTRODUCTION}

This is a paper about multivariate interpolation with radial basis functions, a relatively new, but already well-established and successful technique in the theory of approximation of multivariate functions. Specifically, the purpose of interpolation with radial basis functions is to approximate functions $f: \mathbb{R}^{n} \rightarrow \mathbb{R}$ that are given as data $\{f(\xi)\}$ on a finite set $\Xi=\{\xi\} \subset \mathbb{R}^{n}$ of distinct points ("centres") by expressions

$$
s(x)=\sum_{\xi \in \Xi} \lambda_{\xi} \phi(\|x-\xi\|), \quad x \in \mathbb{R}^{n} .
$$

In (1.1), $\phi: \mathbb{R}_{+} \rightarrow \mathbb{R}$ is a continuous, univariate function, called a radial basis function, and $\|\cdot\|$ is in this paper always the Euclidean norm on $n$-dimensional real space $\mathbb{R}^{n}$. The $\lambda_{\xi}$ are real coefficients, usually fixed through interpolation conditions at the centres

$$
\left.s\right|_{\Xi}=\left.f\right|_{\Xi}
$$

and we note that there are as many coefficients, i.e. degrees of freedom, in (1.1) as there are conditions in the interpolation requirements in (1.2). In order that these conditions may give rise to a unique interpolant (1.1), the symmetric matrix $A=$

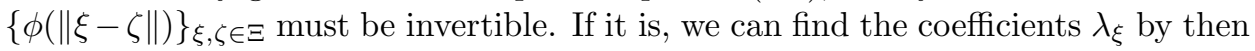
solving the associated linear system.

Many examples of radial basis functions have been put forward that fulfill this nonsingularity requirement, and we intent to propose further useful ones that do as well. The advantage in using radial basis functions is generally that they provide interpolants irrespective of the geometry of the centres $\Xi$ and for any dimension $n$, whereas for instance polynomial interpolation or even piecewise polynomials are

Received by the editor January 7, 1999.

2000 Mathematics Subject Classification. Primary 41A05, 41A15, 41A25, 41A30, 65D05, $65 \mathrm{D} 15$. 
no longer available or feasible in practical applications when $n$ is very large or the geometry of $\Xi$ is complicated.

The main virtue of radial basis functions, namely being readily available for interpolation even in high dimensional settings, is important, for instance, in neural network applications, where the dimension of the underlying approximation problem which we have to solve is often very large. Another attractive feature is that many of them are related to elliptic differential operators, in that they are Green's functions to, say, Laplace or other radially invariant operators. The thin-plate spline $\phi(r)=r^{2} \log r$ is a prime example for that, as it is a fundamental solution of the two-dimensional biharmonic operator. In contrast to the functions we present here, it is not of compact support, but several of those radial functions we deal with in the present article are closely related to the thin-plate spline. Many good reviews have been written on this subject, and we especially mention the review (Powell, 1992) as a good, comprehensive introduction to radial functions.

Other currently used choices of $\phi$ that give good, accurate approximations if the centres are close enough together include the multiquadric radial function $\phi(r)=$ $\sqrt{r^{2}+c^{2}}, c$ being a nonnegative parameter, and Gaussians $\phi(r)=e^{-c^{2} r^{2}}, c$ a positive parameter. Since the Gaussian radial basis function is so well localised in space, the parameter $c$ in it should normally be dependent on the distances of points within $\Xi$; otherwise approximations are unlikely to deliver useful results, especially if the parameter is much too large in comparison with an average distance between the centres $\xi$.

As is required, multiquadrics give invertible matrices $A$ for all dimensions, all sets of distinct centres and all parameters, as do indeed the Gaussians, but the latter have the additional strong advantage that they give a positive definite, essentially banded interpolation matrix. In fact, the banded structure of the matrix $A$ becomes more dominant if the parameter $c$ in the Gaussian radial function is large; but this parameter pits locality against the accuracy of the approximation. We will meet this typical trade-off between locality and quality of the approximant in this paper as well. Furthermore, radial functions of compact support are now being proposed that also give positive definite matrices $A$ and have genuinely banded interpolation matrices. Of such nature are those ones we will discuss in the present paper. We shall also investigate their further properties, such as approximational efficacy, in some detail.

Early examples of radial functions with compact support that have a simple piecewise polynomial structure are due to $\mathrm{Wu}$ (1995), one of the first papers on compactly supported radial functions, further discussed in Schaback and Wu (1996), and to Wendland (1995 and 1998), who established several of their special properties, such as certain optimality facts about their degree and smoothness. The latter two papers are, in the present author's opinion, particularly pleasant due to the simple form of the radial functions. Our class of radial basis functions stems from (Buhmann, 1998), and we enlarge it here, so as to give smoother radial functions with better approximation orders and to include those in (Wendland, 1995) in some sense. They are closely related to the so-called multiply monotone radial basis functions discussed in (Micchelli, 1986), (Buhmann, 1988) and (Buhmann and Micchelli, 1991), the latter multiply monotone ones, however, being of global support. 
The article has three further sections. The next one introduces the class of radial functions we discuss here and proves positive definiteness of the interpolation matrix $A$. The third section considers its approximation properties, while the final section draws comparisons with other known classes of radial functions with compact support.

\section{Definition of the Class}

In this section the class of radial basis functions which we shall study in the article is introduced and their salient properties are established. It contains compactly supported radial functions such as the two-dimensional $(n=2)$ one for example which is three times continuously differentiable

$$
\phi(r)= \begin{cases}\frac{112}{45} r^{\frac{9}{2}}+\frac{16}{3} r^{\frac{7}{2}}-7 r^{4}-\frac{14}{15} r^{2}+\frac{1}{9}, & \text { if } 0 \leq r \leq 1, \\ 0, & \text { if } r>1 .\end{cases}
$$

Here $r$ is for $\|x\|$, with $x \in \mathbb{R}^{n}$. Moreover, in what follows, $n$ is always the dimension of the multivariate real space we work in.

At conferences in Montecatini (June, 1998) and Eilat (September, 1998) the following definition and theorem were given and partially proved. In the subsequent theorem, $\lceil 2 \alpha\rceil$ denotes the least integer $\geq 2 \alpha$. The notation $(\cdot)_{+}$stands for the truncated power function.

Definition and Theorem 1. Let $0<\delta \leq \frac{1}{2}, \varrho \geq 1$ be reals, $g(\beta):=\left(1-\beta^{\delta}\right)_{+}^{\varrho}$, and suppose $\lambda \neq 0$ and $\alpha$ are also real quantities with

$$
\lambda \in\left\{\begin{array}{lll}
\left(-\frac{1}{2}, \infty\right), & -1<\alpha \leq \min \left[\frac{1}{2}, \lambda-\frac{1}{2}\right], & \text { if } n=1, \text { or } \\
{[1, \infty),} & -\frac{1}{2}<\alpha \leq \frac{1}{2} \lambda, & \text { if } n=1, \text { and } \\
\left(-\frac{1}{2}, \infty\right), & -1<\alpha \leq \min \left[\frac{1}{2}\left(\lambda-\frac{1}{2}\right), \lambda-\frac{1}{2}\right], & \text { if } n=2, \text { and } \\
{[0, \infty),} & -1<\alpha \leq \frac{1}{2}(\lambda-1), & \text { if } n=3, \text { and } \\
\left(\frac{1}{2}(n-5), \infty\right), & -1<\alpha \leq \frac{1}{2}\left(\lambda-\frac{1}{2}(n-1)\right), & \text { if } n>3 .
\end{array}\right.
$$

Then the radial basis function

$$
\phi(\|x\|)=\int_{0}^{\infty}\left(1-\|x\|^{2} / \beta\right)_{+}^{\lambda} \beta^{\alpha} g(\beta) d \beta, \quad x \in \mathbb{R}^{n},
$$

has a strictly positive Fourier transform and therefore gives rise to positive definite interpolation matrices A. Moreover, $\phi(\|\cdot\|) \in C^{1+\lceil 2 \alpha\rceil}\left(\mathbb{R}^{n}\right)$.

We note that the class in (Buhmann, 1998) is for $\alpha=0$ contained in the above. The claim in the theorem that $\phi$ having a positive Fourier transform means $A$ is positive definite, is known as

Bochner's Theorem. If the Fourier transform of an integrable continuous $F$ : $\mathbb{R}^{n} \rightarrow \mathbb{R}$ is positive, then the symmetric matrix with entries $F(\xi-\zeta), \xi, \zeta \in \Xi$, is positive definite for all finite sets of distinct points $\Xi \subset \mathbb{R}^{n}$. Conversely, if the aforementioned matrix is positive definite for all finite $\Xi$ and $F$ is continuous, then $F$ must be the Fourier transform of a finite Borel measure.

For Bochner's theorem, see (Steward, 1976). We remark also that (2.1) is such that $\phi(\sqrt{r})$ is $\lambda+1$ times monotone if $\lambda \in \mathbb{N}$, according to the following definition 
and theorem from (Williamson, 1956) and because $g \geq 0$. Only a change of variables in (2.1) is required for showing that.

Definition and Theorem 2. Let $\lambda>1$ be an integer. A function $h: \mathbb{R}_{>0} \rightarrow \mathbb{R}$ is $\lambda$ times monotone if $(-1)^{j} h^{(j)}$ is nonnegative, nonincreasing and convex for all $j<\lambda-1$. A necessary and sufficient condition that $h$ be $\lambda$ times monotone is that there is a nondecreasing measure $\mu$ which is bounded below with

$$
h(t)=\int_{0}^{\infty}(1-t \beta)_{+}^{\lambda-1} d \mu(\beta), \quad t>0 .
$$

The final remark we make before embarking on the proof of Theorem 1 is that, under certain additional conditions (Micchelli, 1986), (Buhmann, 1988), which we do not spell out in this paper, multiply monotone functions, which also may be of global support, are conditionally positive definite of order $k$ : this is so if $(-1)^{k} \phi^{(k)}$ is $\lambda$ times monotone but not constant and $\lambda=\left[\frac{1}{2} n+2-k\right]>0$ (here and below $[\cdot]$ denotes the integer part or the so-called Gauss-bracket).

We shall now prove Theorem 1. It is obvious that $\phi(\|\cdot\|)$ is integrable as a function on $\mathbb{R}^{n}$, because it is supported in the unit ball and continuous. Therefore it has a continuous (in fact analytic) Fourier transform, which we shall compute and whose positivity we must show. The letter $J$ below denotes the Bessel function (Gradsteyn and Ryzhik, 1980, p. 951) which comes from Fourier transforming the characteristic function of the Euclidean unit ball. The following computation of $\hat{\phi}$ uses standard means to compute Fourier transforms of radially symmetric, integrable functions: By (Stein and Weiss, 1971, p. 137)

$$
\hat{\phi}(\|x\|)=(2 \pi)^{n / 2}\|x\|^{1-n / 2} \int_{0}^{\infty} \int_{0}^{\infty}\left(1-s^{2} / \beta\right)_{+}^{\lambda} s^{n / 2} J_{n / 2-1}(s\|x\|) d s \beta^{\alpha} g(\beta) d \beta,
$$

$x \in \mathbb{R}^{n}$, where we point out that we have transformed the $n$-variate, rotationally invariant function $\phi(\|\cdot\|)$ to get the rotationally invariant multivariate Fourier transform $\hat{\phi}(\|\cdot\|)$ with univariate radial part $\hat{\phi}$. Now, by integrating the truncated power against the Bessel function, see (Gradsteyn and Ryzhik, 1980, p. 688), and by denoting the standard Gamma-function by $\Gamma$, we transform the above into

$$
\hat{\phi}(\|x\|)=(2 \pi)^{n / 2} 2^{\lambda} \Gamma(\lambda+1)\|x\|^{-\lambda-n / 2} \int_{0}^{\infty} J_{\lambda+n / 2}(\|x\| \sqrt{\beta}) \beta^{n / 4-\lambda / 2+\alpha} g(\beta) d \beta,
$$

$x \in \mathbb{R}^{n}$, which becomes, by $t^{2}=\|x\|^{2} \beta$,

$$
(2 \pi)^{n / 2} 2^{\lambda+1} \Gamma(\lambda+1)\|x\|^{-n-2-2 \alpha} \int_{0}^{\infty} J_{\lambda+n / 2}(t) t^{n / 2-\lambda+1+2 \alpha} g\left(t^{2}\|x\|^{-2}\right) d t .
$$

Finally, by including our particular weight function $g$ we get the radial basis function

$$
\begin{aligned}
\hat{\phi}(\|x\|)= & (2 \pi)^{n / 2} 2^{\lambda+1} \Gamma(\lambda+1)\|x\|^{-n-2-2 \delta \varrho-2 \alpha} \\
& \times \int_{0}^{\infty} J_{\lambda+n / 2}(t) t^{n / 2-\lambda+1+2 \alpha}\left(\|x\|^{2 \delta}-t^{2 \delta}\right)_{+}^{\varrho} d t .
\end{aligned}
$$

We claim that (2.3) is positive for all arguments. To this end we take from (Misiewicz and Richards, 1994, p. 599) the following auxiliary result: 
Proposition 3. Let $0<\delta \leq \frac{1}{2}$, $\varrho \geq 1$ be real quantities. Then the Hankel transform

$$
\int_{0}^{x}\left(x^{2 \delta}-t^{2 \delta}\right)^{\varrho} t^{\mathbf{a}} J_{\mathbf{b}}(t) d t
$$

is positive for all positive reals $x$ if $\mathbf{a}$ and $\mathbf{b}$ satisfy any of the following conditions:

(i) $\frac{3}{2}=\mathbf{a} \leq \mathbf{b}$,

(ii) $-\frac{1}{2} \leq \mathbf{a}=\mathbf{b} \leq \frac{3}{2}$,

(iii) $\frac{1}{2}=\mathbf{a}<\mathbf{b}$,

(iv) $\mathbf{a}=\mathbf{a}_{0}-\delta_{0}, \mathbf{b}=\mathbf{b}_{0}+\delta_{0}$ if $\mathbf{a}_{0}, \mathbf{b}_{0}$ satisfy any of (i)-(iii) and $\delta_{0}$ is nonnegative.

We remark that Misiewicz and Richards claim only nonnegativity of the integral (2.4), but a close inspection of the inequalities in (Gasper, 1975, pp. 868f.) they use, namely (1.1) and (1.5), shows that the integral is actually strictly positive.

Now, for the application of Proposition 3, we first take $n>3$ in the proof of Theorem 1. Then, choosing in Proposition 3 the parameters $\mathbf{a}=\frac{1}{2} n-\lambda+1+2 \alpha$, $\mathbf{b}=\lambda+\frac{1}{2} n, \mathbf{a}_{0}=\frac{1}{2} n-\lambda+1+2 \alpha+\delta_{0}, \mathbf{b}_{0}=\frac{1}{2} n+\lambda-\delta_{0}$, and $\delta_{0}=\frac{1}{2}+\lambda-\frac{1}{2} n-2 \alpha$, we get the required result for $n>3$ from (iv) and (i) in our proposition. For that, we note that $\delta_{0}$ must be nonnegative, and this requirement leads to the condition on $\alpha$ in the statement of our theorem, i.e. $2 \alpha \leq \lambda-\frac{1}{2}(n-1)$. The additional requirement in (i) that $\mathbf{b}_{0} \geq \frac{3}{2}$ means in this context $n+2 \alpha \geq 2$, which is true under the hypotheses of the theorem when $n \geq 4$. When $n=2$ or $n=3$, we apply (iv) and (i) again to get the admissible ranges $0 \leq \alpha \leq \frac{1}{2}\left(\lambda-\frac{1}{2}\right), \lambda \geq \frac{1}{2}$, and $-\frac{1}{2} \leq \alpha \leq \frac{1}{2}(\lambda-1), \lambda \geq 0$, respectively.

However, we then supplement the admissible ranges by using (ii) in addition (i), because (ii) admits the following parameters, with the same analysis as before. For $n=2,-1<\alpha \leq 0, \alpha \leq \lambda-\frac{1}{2}$ and $\lambda>-\frac{1}{2}$, and, for $n=3,-1<\alpha \leq-\frac{1}{2}$, $\alpha \leq \lambda-\frac{1}{2}$ and $\lambda>-\frac{1}{2}$. In both cases, $\delta_{0}=\lambda-\alpha-\frac{1}{2}$. Together with the results from the application of (i) and our earlier results for $n>3$, we get the assertion of the theorem for all $n>1$.

For $n=1$, we apply (iv) and (ii) with the choice $\mathbf{a}_{0}=\mathbf{b}_{0}=\alpha+1$ and $\delta_{0}=$ $\lambda-\alpha-\frac{1}{2}$. The latter must be nonnegative, and this requirement yields the condition $\alpha \leq \lambda-\frac{1}{2}$. That is fulfilled as required in the statement of the theorem, but, for the application of the bounds in (ii), we have to require additionally that $-\frac{1}{2} \leq \mathbf{a}_{0} \leq \frac{3}{2}$, which leads to the extra condition $-\frac{3}{2} \leq \alpha \leq \frac{1}{2}$, satisfied by the conditions of the theorem.

When conditions (iv), (i) and (iii) of Proposition 3 are used, we get the parameter $\delta_{0}=\lambda-2 \alpha-1$ in the latter case. Item (i) leads to the requirements $\lambda \geq 1$ and $\frac{1}{2} \leq$ $\alpha \leq \frac{1}{2} \lambda$, whereas item (iii) and the nonnegativity of $\delta_{0}$ lead to $-\frac{1}{2}<\alpha \leq \frac{1}{2}(\lambda-1)$. We may combine these conditions with the condition stated in the theorem, because, when $-\frac{1}{2}<\alpha<\frac{1}{2}$ and therefore we are out of the range of the admitted $\alpha$ from item (i), then (ii) applies and allows the missing range. Indeed, item (ii) with $\delta_{0}=\lambda-\alpha-\frac{1}{2}$ and $\mathbf{a}_{0}=\mathbf{b}_{0}=\alpha+1$ makes $-\frac{3}{2} \leq \alpha \leq \frac{1}{2}$ admissible as long as $\alpha \leq \lambda-\frac{1}{2}$.

Therefore we have established that (2.3) is always positive under the conditions stated in Theorem 1.

The next fact we need to prove is the smoothness of our radial function as a multivariate, radially symmetric function. There are various ways to do this, and the simplest one is to observe that (2.1) becomes, through a change of variables 
and using the fact that truncated powers are in the integrand, the expression

$$
\phi(\|x\|)=\|x\|^{2 \alpha+2} \int_{1}^{\|x\|^{-2}}\left(1-\beta^{-1}\right)^{\lambda} \beta^{\alpha}\left(1-\beta^{\delta}\|x\|^{2 \delta}\right)^{\varrho} d \beta .
$$

Now, the expression $\|x\|^{2 \alpha+2}$ is clearly in the smoothness class required in the statement of the theorem. Moreover, we note that we only have to establish the differentiability in a neighbourhood of zero and near $\|x\|=1$, since elsewhere the radial function we consider is of course infinitely smooth.

For the former range, we expand in (2.5) the two binomial expressions in the integrand, their arguments always being less than one in modulus, in infinite series, integrate them term by term explicitly, and verify by inspection that multiples of $\|x\|^{2 \alpha+2}$ are indeed the terms of lowest smoothness that occur in the series, all others being of the form $\|x\|^{2 \alpha+2+2 \delta j}, j=1,2,3, \ldots$, or even powers of $\|x\|$, sometimes multiplied by a $\log \|x\|$ term. Therefore, the differentiability assertion at zero is justified.

For the differentiability at any $x$ with $\|x\|=1$, we note that, by the mean value theorem for integrals, the integrand being always nonnegative, there is a $\tilde{\beta} \in\left[\|x\|^{2}, 1\right]$ such that for all $0 \ll\|x\|<1$

$$
\begin{aligned}
\phi(\|x\|) & =\int_{\|x\|^{2}}^{1}\left(1-\|x\|^{2} / \beta\right)^{\lambda} \beta^{\alpha}\left(1-\beta^{\delta}\right)^{\varrho} d \beta \\
& =\left(1-\|x\|^{2} / \tilde{\beta}\right)^{\lambda}\left(1-\tilde{\beta}^{\delta}\right)^{\varrho} \int_{\|x\|^{2}}^{1} \beta^{\alpha} d \beta .
\end{aligned}
$$

By (2.6), where the rightmost integral can be evaluated as

$$
\left(1-\|x\|^{2 \alpha+2}\right) /[\alpha+1]
$$

the radial function is at least $\lfloor\lambda+\varrho+1\rfloor \geq \lambda+1$ times continuously differentiable at any $x$ with $\|x\|=1$. Here, $\lfloor\cdot\rfloor$ denotes the largest integer less than the argument. The number $\lambda+1$, however, is always at least $2 \alpha+2 \geq\lceil 2 \alpha\rceil+1$ when $n>2$ by the restrictions on the ranges of $\alpha$ in the statement of the theorem, because $\lambda$ is at least $2 \alpha+\frac{1}{2}(n-1)$.

In the remaining cases $n=1$ and $n=2$ we observe that it is straightforward to argue for $\varrho>1$, or when $\lambda$ is not integral, as follows, where we use the Gauss-bracket as before: $\lfloor\lambda+\varrho+1\rfloor \geq[\lambda]+2 \geq \min \left(\left[2 \alpha+\frac{1}{2}\right]+2,[2 \alpha]+2,\left[\alpha+\frac{1}{2}\right]+2\right) \geq\lceil 2 \alpha\rceil+1$, where in the final item in the minimum we also use that $\alpha \leq \frac{1}{2}$ in that event anyway, by the conditions on $\alpha$ of Theorem 1 .

The remaining case is when $n=1$ or $n=2$ and $\lambda$ integral, $\varrho=1$, which we treat separately. Thus

$$
\lfloor\lambda+\varrho+1\rfloor=\lambda+1
$$

When $n=2$, we know from the hypotheses about $\alpha$ that $(2.7)$ is at least $2 \alpha+\frac{3}{2}$. If $\alpha-[\alpha] \geq \frac{1}{2}$, then this is always at least $\lceil 2 \alpha\rceil+1$. Otherwise (2.7) is at least $\lceil 2 \alpha\rceil+1$, because $\lambda$ is integral.

We can argue similarly for $n=1$. We still have integral $\lambda$. Thus either by the conditions on $\alpha$ of Theorem 1 we have $\lambda+1 \geq 2 \alpha+1$ and indeed $\lambda+1 \geq\lceil 2 \alpha\rceil+1$; or, $\lambda+1 \geq \alpha+\frac{3}{2}$, which implies $\lambda+1 \geq\lceil 2 \alpha\rceil+1$ so long as $\alpha \leq \frac{1}{2}$, demanded explicitly in the statement of the theorem. 
There is another way to prove smoothness if $\alpha$ and $\varrho$ satisfy some small extra conditions. We demonstrate that alternative proof, which establishes smoothness of the function via the decay properties of its Fourier transform, too, because it will be extremely helpful to us later in the convergence proof in the next section. To this end, we require the following Lemma 4 in (Buhmann, 1989).

Lemma 4. Suppose we are given $q \in \mathbb{N}$ and $\sigma \in C^{q}\left(\mathbb{R}_{>0}\right)$, and suppose that we have the short asymptotic expansion near the origin

$$
\sigma^{(\ell)}(t)=\frac{d^{\ell}}{d t^{\ell}} d_{0} t^{e_{0}}+O\left(t^{e_{1}-\ell}\right), \quad t \rightarrow 0,0 \leq \ell \leq q,
$$

with real $d_{0}$ and reals $e_{0}<e_{1}$, where $e_{0}>-\nu-1$ and where $\nu>0, q \geq 2 e_{1}+\nu+2$. Let

$$
\hat{\phi}(r)=r^{-\nu} \int_{0}^{\infty} J_{\nu}(r \beta) \mathbf{f}(\beta) d \beta, \quad r>0
$$

where

$$
\mathbf{f}(\beta)=\beta^{\nu+1} \sigma\left(\beta^{2}\right), \quad \beta>0,
$$

be well defined for every positive $r$. Then for any

$$
0<\gamma<2 \min \left(e_{0}+\nu+1, e_{1}-e_{0}, \frac{1}{2} \nu\right)
$$

it satisfies the short asymptotic expansion

$$
\hat{\phi}(r)=\frac{\Gamma\left(\nu+e_{0}+1\right) d_{0}}{2^{\nu+1} \Gamma\left(-e_{0}\right)}\left(\frac{1}{2} r\right)^{-2 e_{0}-2 \nu-2}+O\left(r^{-2 e_{1}-2 \nu-2+\gamma}\right), \quad r \rightarrow \infty .
$$

We apply the lemma to (2.2) by choosing $\nu=\lambda+\frac{1}{2} n$, which is positive by hypothesis on $\lambda, e_{0}=\alpha-\lambda$ and $\sigma(\beta)=\beta^{\alpha-\lambda} g(\beta)$. The condition $e_{0}>-\nu-1$ is satisfied if we demand $\alpha>-1$, because this immediately implies $e_{0}=\alpha-\lambda>$ $-\lambda-\frac{1}{2} n-1$. Further we let $e_{1}=\alpha-\lambda+\delta>e_{0}$. Therefore the choice $q=\lfloor\varrho\rfloor \geq 1$ requires

$$
\lfloor\varrho\rfloor \geq 1 \geq 2 e_{1}+\nu+2=2 \alpha+2 \delta-\lambda+\frac{1}{2} n+2 .
$$

Here, we still use $\lfloor\varrho\rfloor$ as the largest integer $<\varrho$. Recalling $\delta \leq \frac{1}{2}$, it is sufficient for this that

$$
\alpha \leq \frac{1}{2}\left(\lambda-\frac{1}{2} n-3+\lfloor\varrho\rfloor\right)
$$

or indeed, together with the additional requirement $\alpha>-1$,

$$
-1<\alpha \leq \frac{1}{2}\left(\lambda-\frac{1}{2} n-2\right)
$$

Therefore the extra conditions we require to apply Lemma 4 are $(2.9)$ and $\varrho>1$ and $\lambda>\frac{1}{2} n$, the penultimate demand being to ensure that $\lfloor\varrho\rfloor \geq 1$.

We obtain that the Fourier transform of $\phi(\|\cdot\|)$ has a radial part $\hat{\phi}$ that is, of course, continuous and bounded at the origin, and also satisfies the asymptotic estimate $\hat{\phi}(r) \sim r^{-n-2-2 \alpha}$ plus higher order terms (that is, higher order in modulus of the exponent) at infinity. Here it is important to notice that the first term in the expansion (2.8) is nonzero, because $1 / \Gamma\left(-e_{0}\right)$ does not vanish due to $\alpha<\lambda$ and hence $e_{0}<0$. 
Therefore, the multivariate function $\phi(\|\cdot\|)$ is at least $1+\lceil 2 \alpha\rceil$ times continuously differentiable, because the partial derivatives of total order $\ell$ have absolutely integrable Fourier transforms with asymptotic decay rate $r^{-n-2 \alpha-2+\ell}$ (e.g. Stein and Weiss, 1971, p. 4) and because $2 \alpha+2-1-\lceil 2 \alpha\rceil$ is positive.

Two examples that give twice and three-times continuously differentiable functions, respectively, in three dimensions and two dimensions are as follows. The choices $\alpha=\delta=\frac{1}{2}, \varrho=1$, and $\lambda=2$, give $(n=3)$

$$
\phi(r)=2 r^{4} \log r-\frac{7}{2} r^{4}+\frac{16}{3} r^{3}-2 r^{2}+\frac{1}{6},
$$

while the choices $\alpha=\frac{3}{4}, \delta=\frac{1}{2}, \varrho=1$, and $\lambda=2$ provide for $n=2$

$$
\phi(r)=\frac{112}{45} r^{\frac{9}{2}}+\frac{16}{3} r^{\frac{7}{2}}-7 r^{4}-\frac{14}{15} r^{2}+\frac{1}{9} .
$$

Both cases apply for $0 \leq r \leq 1$; otherwise, $\phi(r)=0$.

In fact the results of Misiewicz and Richards are even more general than those we have used so far, because in the proof of Theorem 1 we can replace

$$
\|x\|^{2 \delta \varrho}\left(1-(t /\|x\|)^{2 \delta}\right)_{+}^{\varrho}=\left(\|x\|^{2 \delta}-t^{2 \delta}\right)_{+}^{\varrho}
$$

by the expression $\|x\|^{2 \delta \varrho} \Phi((t /\|x\|))$ with any twice monotone function $\Phi$ that vanishes at 1 . In other words, we replace our $g(\beta)$ in the statement of the theorem above by $\Phi(\sqrt{\beta})$. We note that it is sufficient if we specify $\Phi$ on $(0,1]$ and then continue it as zero on $(1, \infty)$.

An example for this generalisation is $\Phi(t)=t-1-\log t, 0 \leq t \leq 1, \Phi(t)=0$ otherwise. For $\alpha=\frac{1}{2}$ and $\lambda=n=1$ it gives the radial function

$$
\phi(r)=\frac{1}{18}-r^{2}+\frac{4}{9} r^{3}+\frac{1}{2} r^{4}-\frac{4}{3} r^{3} \log r .
$$

This positive definite radial basis function of compact support is twice continuously differentiable.

\section{Convergence Results}

In this section we investigate some of the convergence properties of the radial functions introduced above. We let $\Omega \subset \mathbb{R}^{n}$ be a compact domain and denote by $D^{-\kappa} L^{2}\left(\mathbb{R}^{n}\right)$ the space of all distributions whose partial derivatives of total order $\kappa$ are square-integrable, $\kappa$ not necessarily being an integer (see also Stein and Weiss, 1971). Under the stricter condition that the domain has a Lipschitz continuous boundary which satisfies an interior cone condition, the proof of a theorem that has a stronger convergence result can be found in (Wendland, 1997), but we explain and comment on the short proof here nonetheless because some techniques which are fundamental to the theory of radial functions and important to this paper are involved, and because we do not require the aforementioned stronger properties of $\Omega$.

Theorem 5. Let $\phi$ be as in Theorem 1, with the extra condition in (2.9) and in the sentence thereafter in place. Let $\Xi$ be a finite set in $\Omega$ with the distance

$$
h=\max _{x \in \Omega} \min _{\xi \in \Xi}\|x-\xi\| .
$$


Let $s$ be the interpolant (1.1) to $f \in L^{2}\left(\mathbb{R}^{n}\right) \cap D^{-n / 2-1-\alpha} L^{2}\left(\mathbb{R}^{n}\right)$, scaled of the form (for a positive scaling parameter $\eta$ )

$$
s(x)=\sum_{\xi \in \Xi} \lambda_{\xi} \phi\left(\eta^{-1}\|x-\xi\|\right), \quad x \in \mathbb{R}^{n},
$$

with the interpolation conditions $\left.(s-f)\right|_{\Xi}=0$ satisfied. Then the uniform convergence estimate

$$
\|f-s\|_{\infty, \Omega} \leq C h^{1+\alpha}\left(\eta^{-n / 2-1-\alpha}+1\right)
$$

holds for $h \rightarrow 0$ and positive bounded $\eta$, the positive constant $C$ being independent of both $h$ and $\eta$. Here $\|\cdot\|_{\infty, \Omega}$ denotes the Chebyshev norm on $\Omega$.

We observe that an $f$ which satisfies the conditions of the theorem is also continuous by the Sobolev embedding theorem, as $\alpha>-1$. We note also that it is suitable to scale the radial function by $\eta$, because its support should depend on the geometry of $\Xi$, the wish being that there are not too many points in the support of each shifted and scaled radial function so that the interpolation matrix $A$ is sparse. In other words, $\eta$ should shrink as $h \rightarrow 0$. Our error estimate above, however, requires that $\eta$ does not go to zero too fast with $h \rightarrow 0$, in order to retain convergence, so there is a trade-off between the sparsity of the matrix and convergence according to Theorem 5. This is strongly reminiscent of the so-called uncertainty principle introduced by Schaback, when he showed that there is a trade-off between accuracy of interpolation and condition numbers of radial function interpolation matrices for a large class of radial basis functions.

Proof. The first part of the proof is a standard argument using the so-called native spaces associated with radial basis functions and the power functions - see (Wu and Schaback, 1993), where the power functions are still called "Kriging functions", power function being the name for the same object more frequently in use now; see also that paper for further references. To see how this argument works, it is, first of all, necessary to explain what the "native space" is. Our native space here is, in short, a reproducing kernel Hilbert space $X$, whose reproducing kernel is $\phi_{\eta}(\|\cdot\|)$, with $\phi_{\eta}$ as a convenient notation for $\phi(\cdot / \eta)$. Now, the space $X$ is induced by the inner product

$$
(f, g)=\frac{1}{(2 \pi)^{n}} \int_{\mathbb{R}^{n}} \frac{1}{\hat{\phi}_{\eta}(\|t\|)} \hat{f}(t) \overline{\hat{g}(t)} d t,
$$

which is well-defined for all suitable $f$ and $g$, because of the positivity everywhere of the $\hat{\phi}_{\eta}$ in the weight. The norm associated with this inner product is

$$
\|f\|_{\phi_{\eta}}=\sqrt{(f, f)} .
$$

The native space $X$ therefore consists of all distributions $f$ with finite Hilbert space norm (3.1).

The reproducing kernel property is especially easy to show, because our compactly supported radial function here is absolutely integrable and positive definite due to the positivity of its Fourier transform. Briefly, for any $f \in X$ we have almost everywhere (and thus everywhere, since all functions we use are continuous) the identity $\left(f, \phi_{\eta}(\|x-\cdot\|)\right)=f(x)$, because the Parseval-Plancherel formula 
implies almost everywhere

$$
\begin{aligned}
\left(f, \phi_{\eta}(\|x-\cdot\|)\right) & =\frac{1}{(2 \pi)^{n}} \int_{\mathbb{R}^{n}} \frac{1}{\hat{\phi}_{\eta}(\|t\|)} \hat{f}(t) e^{i x \cdot t} \overline{\hat{\phi}_{\eta}(\|t\|)} d t \\
& =\frac{1}{(2 \pi)^{n}} \int_{\mathbb{R}^{n}} \hat{f}(t) e^{i x \cdot t} d t=f(x) .
\end{aligned}
$$

Now, the work in (Wu and Schaback, 1993) provides a pointwise local estimate for the error on the domain $\Omega$, namely

$$
|f(x)-s(x)| \leq C(h / \eta)^{1+\alpha}\|f\|_{\phi_{\eta}} .
$$

In order to get the required bound for this proof, we have to take the uniform norm of this bound over all $x \in \Omega$ :

$$
\|f-s\|_{\infty, \Omega} \leq C(h / \eta)^{1+\alpha}\|f\|_{\phi_{\eta}},
$$

where $\|\cdot\|_{\infty, \Omega}$ still denotes the $L^{\infty}$ norm restricted to the domain $\Omega$. The $h$ dependent factor in (3.3) comes, as shown in (Wu and Schaback, 1993), from the bound on the power functional, and the last factor comes from the native space norm that we encountered in (3.1).

Next, the rightmost factor of (3.3) can be bounded as follows, because $\hat{\phi}(0)$ is a positive, finite number, whence we have the relation $\hat{\phi}(r) \sim(1+r)^{-n-2-2 \alpha}$ for all nonnegative $r$, recalling from the Lemma 4 the short asymptotic expansion $\hat{\phi}(r) \sim r^{-n-2-2 \alpha}, r \rightarrow \infty:$

$$
\begin{aligned}
(2 \pi)^{n} \eta^{n}\|f\|_{\phi_{\eta}}^{2} & =\int_{\mathbb{R}^{n}} \frac{1}{\hat{\phi}(\eta\|t\|)}|\hat{f}(t)|^{2} d t \\
& \leq C\|f\|_{2}^{2}+C \eta^{n+2+2 \alpha} \int_{\mathbb{R}^{n}}\|t\|^{n+2+2 \alpha}|\hat{f}(t)|^{2} d t .
\end{aligned}
$$

Note that this implies that our reproducing kernel Hilbert space $X$ is a superset of $L^{2}\left(\mathbb{R}^{n}\right) \cap D^{-n / 2-1-\alpha} L^{2}\left(\mathbb{R}^{n}\right)$.

By virtue of (3.3), this gives in total the error estimate

$$
\|f-s\|_{\infty, \Omega} \leq C(h / \eta)^{1+\alpha}\left(\eta^{-n / 2}+\eta^{1+\alpha}\right),
$$

with an $f$-dependent constant $C$. Finally, to get the assertion of the theorem, we have to combine the powers of $h$ and $\eta$ in the above display. This gives the required result. The theorem is proved.

To finish this section we add a few remarks about the $\ell^{2}$ condition numbers of the matrix $A$, in particular as functions of the minimal separation distance

$$
q_{\Xi}=\frac{1}{2} \min _{\xi \neq \zeta}\|\xi-\zeta\|>0
$$

of the set $\Xi$ of centres, because they are highly relevant to the numerical stability of the linear systems related to the interpolation problem. Standard arguments from the seminal work on condition numbers of interpolation matrices of Narcowich and Ward (1991) that have nothing to do with our particular choice of radial basis function show that

$$
\|A\|_{2} \leq\left(\frac{4}{q_{\Xi}}\right)^{n}
$$


Moreover, from Wendland (1998) we take Theorem 4.1, which shows in our context that

$$
\left\|A^{-1}\right\|_{2} \leq C q_{\Xi}^{-2-2 \alpha}
$$

as a consequence of the decay of $\hat{\phi}$ as established in the proof of Theorem 1 with the aid of Lemma 4. Thus the least squares condition number is $\operatorname{cond}_{2}(A)=$ $O\left(q_{\Xi}^{-n-2-2 \alpha}\right)$. Whenever the interpolation matrix uses $\phi_{\eta}$ instead of $\phi$ in the sense of the previous theorem, $q_{\Xi}$ must be replaced by $\eta^{-1} \cdot q_{\Xi}$ in the above estimates.

\section{COMPARISON WITH OTHER COMPACTLY SUPPORTED RADIAL BASIS FUNCTIONS}

In this section we show how the functions introduced by Wendland (1995) and $\mathrm{Wu}(1995)$ are related to those discussed in this work. For this it is relevant to use the "operators on radial functions" studied in Schaback and Wu (1996). Specifically, we employ the differentiation operator for differentiable $f$

$$
\mathcal{D} f(r)=-\frac{1}{r} f^{\prime}(r), \quad r \geq 0 .
$$

When this is applied to our radial functions (2.1), it gives, for $\delta=\frac{1}{2}$ and any integral $\lambda$,

$$
\mathcal{D}^{\lambda} \phi(r)=\Gamma(\lambda+1) 2^{\lambda} \int_{r^{2}}^{1} \beta^{\alpha-\lambda}(1-\sqrt{\beta})^{\varrho} d \beta, \quad 0 \leq r \leq 1,
$$

and therefore, by one further application of the differentiation operator and explicit evaluation,

$$
\mathcal{D}^{\lambda+1} \phi(r)=\Gamma(\lambda+1) 2^{\lambda+1} r^{2 \alpha-2 \lambda}(1-r)_{+}^{\varrho}=\Gamma(\lambda+1) 2^{\lambda+1}(1-r)_{+}^{\varrho}, \quad r \geq 0,
$$

for $\alpha=\lambda$. Now let $\lambda=k-1, \varrho=\left[\frac{1}{2} n\right]+k+1$ and recall that, on the other hand, we know that the radial functions $\phi_{n, k}$ of Wendland are such that

$$
\mathcal{D}^{k} \phi_{n, k}(r)=(1-r)_{+}^{\left[\frac{1}{2} n\right]+k+1}, \quad r \geq 0,
$$

- in fact they are defined like that - and that they have a positive Fourier transform by virtue of the positivity of the Fourier transform of $(1-r)_{+}^{\ell}$, for any integral $\ell \geq$ $\left[\frac{1}{2} n\right]+1$, where we are still working in $n$ dimensions. Examples for the Wendland functions are for $n=1$ and $k=2$ the radial function $\phi_{1,2}(r)=(1-r)_{+}^{5}\left(8 r^{2}+5 r+1\right)$, for $n=3$ and $k=3$ the radial function $\phi_{3,3}(r)=(1-r)_{+}^{8}\left(32 r^{3}+25 r^{2}+8 r+1\right)$ and for $n=5$ and $k=1$ the radial function $\phi_{5,1}(r)=(1-r)_{+}^{5}(5 r+1)$.

Furthermore, it is precisely the advantage of the differentiation operator that the positive definiteness of that truncated power is retained when the inverse operator to $\mathcal{D}$, an integration operator, is applied to $(1-r)_{+}^{\left[\frac{1}{2} n\right]+1}$, although the positive definiteness is now in an ambient space of two dimensions lower than before.

Therefore, the functions generated in this fashion can also be derived from the form (2.1), although with a parameter $\alpha$ that does not fulfill the conditions of Theorem 1, so it is a type of continuation. The functions from $\mathrm{Wu}$ (1995) also belong to that class, as they were shown by Wendland to be special cases of his functions. Therefore, with the class of Wendland functions described here containing those introduced by $\mathrm{Wu}$ (1995), our class covers all those. Finally, because we know that all those functions have positive Fourier transforms, we immediately get new results about the positivity of Hankel transforms (2.3) which extend the work by Misiewicz 
and Richards, and this is in spite of the fact that the parameter $\alpha$ here does not yield the conditions of Theorem 1 . We do not elaborate on this, because it is only a matter of inserting the above parameters $\alpha, \varrho, \delta$ and $\lambda$ into (2.1).

\section{ACKNOWLEDGMENTS}

The author thanks Aurelian Bejancu and Oleg Davydov for discussions and pertinent remarks about this work.

\section{REFERENCES}

[1] Buhmann, M.D. (1989) "Cardinal interpolation with radial basis functions: an integral transform approach", in Multivariate Approximation Theory IV, Walter Schempp, Karl Zeller, eds., International Series of Numerical Mathematics Vol. 90, Birkhäuser Verlag (Basel), pp. 41-64. MR 90m:41004

[2] Buhmann, M.D. and C.A. Micchelli (1991) "Multiply monotone functions for cardinal interpolation", Advances in Appl. Math. 12, 358-386. MR 92g:41002

[3] Buhmann, M.D. (1998) "Radial basis functions on compact support", Proceedings of the Edinburgh Math. Society 41, 33-46. [MR 99d:41005

[4] Gasper, G. (1975) "Positive integrals of Bessel functions", SIAM J. Math. Anal. 6, 868-881. MR 52:11144

[5] Gradsteyn, I.S. and I.M. Ryzhik (1980) Table of Integrals, Series, and Products, Academic Press, San Diego. MR 81g:33001

[6] Micchelli, C.A. (1986) "Interpolation of scattered data: distance matrices and conditionally positive definite functions", Constructive Approx. 2, 11-22. MR 88d:65016

[7] Misiewicz, J.K. and D. St.P. Richards (1994) "Positivity of integrals of Bessel functions", SIAM J. Math. Anal. 25, 596-601. MR 95i:33004

[8] Narcowich, F. and J. Ward (1991) "Norms of inverses and condition numbers for matrices associated with scattered data", J. Approx. Th. 64, 84-109. MR 92b:65017

[9] Powell, M.J.D. (1992) "The theory of radial basis function approximation in 1990", in Advances in Numerical Analysis II, W.A. Light, ed., Claredon Press, Oxford, pp. 105-210. MR 95c: 41003

[10] Schaback, R. and Z. Wu (1996) "Operators on radial functions", J. of Computational and Appl. Math. 73, 257-270. MR 97g:42002

[11] Stein, E. and G. Weiss (1971) Introduction to Fourier Analysis on Euclidean Spaces, Princeton University Press, Princeton. MR 46:4102

[12] Stewart, J. (1976) "Positive definite functions and generalizations, an historical survey", Rocky Mountains Math. J. 6, 409-434. MR 55:3679

[13] Wendland, H. (1995) "Piecewise polynomial, positive definite and compactly supported radial basis functions of minimal degree", Advances in Computational Mathematics 4, 389-396. MR 96h:41025

[14] Wendland, H. (1997) "Sobolev-type error estimates for interpolation by radial basis functions", in Surface Fitting and Multiresolution Methods, A. LeMéhauté and L.L. Schumaker, eds., Vanderbilt University Press, Nashville, pp. 337-344. MR 99j:65012

[15] Wendland, H. (1998) "Error estimates for interpolation by radial basis functions of minimal degree, J. Approx. Th. 93, 258-272. MR 99g:65015

[16] Williamson, R.E. (1956) "Multiply monotone functions and their Laplace transforms", Duke Math. J. 23, 189-207. MR 17:1061d

[17] Wu, Z. and R. Schaback (1993) "Local error estimates for radial basis function interpolation of scattered data", IMA J. Numer. Anal. 13, 13-27. MR 93m:65012

[18] Wu, Z. (1995) "Compactly supported positive definite radial functions", Advances in Computational Mathematics 4, 283-292. MR 97g:65031

Lehrstuhl ViII Mathematik, Universität Dortmund, 44221 Dortmund, Germany

Current address: Lehrstuhl Numerik, Justus-Liebig-Universität, Heinrich-Buff-Ring 44, 35392

Giessen, Germany

E-mail address: martin.buhmann@math.uni-giessen.de 